\title{
Uncomputability and physical law
}

\author{
Seth Lloyd \\ Mechanical Engineering, Massachusetts Institute of Technology
}

Computers can do so much that it's easy to forget that they were invented for what they could not do. In his 1937 paper, "On Computable Numbers, with an Application to the Entscheidungsproblem," Alan Turing defined the notion of a universal digital computer (a Turing machine), which became the conceptual basis for the contemporary electronic computer [1]. Turing's goal was to show that there were tasks that even the most powerful computing machine could not perform. In particular, Turing showed that no Turing machine could solve the problem of whether a given Turing machine would halt and give an output when programmed with a given input. In creating a computational analogue to Gödel's incompleteness theorem [2], Turing introduced the concept of uncomputability.

Although Turing's machine was an abstract mathematical construct, his ideas soon found implementation as physical devices [3]. At the end of the 1930s, Konrad Zuse in Germany began building digital computers, first mechanical (the Z-1), and then electrical (the Z-3), and Claude Shannon's 1937 master's thesis showed how digital computers could be constructed using electronic switching circuits [4], a development that presaged the construction of the British code-breaking electronic computer, the Colossus, and the American Mark I. Since computers are physical systems, Turing's uncomputability results show that there are well-formulated questions that can be asked about physical systems whose answers are uncomputable.

That is, there are questions that we can ask about the physical behavior of the universe whose answers are not resolvable by any finite computation. In this chapter, I will investigate the question of how such questions permeate the fabric of physical law. I will show that answers to some of the most basic questions concerning physical law are in fact uncomputable. In particular, one of the primary driving forces of science in general and 
of physics in particular is to create the most succinct formulation of the natural laws. However, straightforward results from algorithmic information theory imply that the most concise formulation of the fundamental laws of nature is in fact uncomputable.

Many physical systems are capable of universal computation: indeed, it is difficult to find an extended system with nonlinear interactions that is not capable of universal computation given proper initial conditions and inputs [5-8]. For such systems, there are always questions that one can ask about their physical dynamics whose answers are uncomputable. One might hope that such questions are sufficiently esoteric that they do not overlap with the usual questions that physicists ask of their systems. I will show that even the answers to such basic questions are often uncomputable. In particular, consider the commonly asked question of whether a physical system has an energy gap - so that its energy spectrum is discrete in the vicinity of its ground state, or whether it is gapless - so that the spectrum is continuous. If the system is capable of universal computation, then the answer to the question of whether it has an energy gap or not is uncomputable.

The uncomputability of the answers to common and basic questions of physics might seem to be a serious hindrance to doing science. To the contrary, the underlying uncomputability of physical law simply puts physicisists in the same position that mathematicians have occupied for some time: many quantities of interest can be computed, but not all. For example, even though the most concise formulation of the underlying laws of physics is uncomputable, short and elegant formulations of physical laws certainly exist. Not knowing in advance whether or not the quantity that one is trying to compute is uncomputable reflects the shared experience of all scientists: one never knows when the path of one's research will become impassible. The underlying uncomputability of physical law simply adds zest and danger to an already exciting quest.

\section{The halting problem}

Start by reviewing the origins of uncomputability. Like Gödel's incompleteness theorem [2], Turing's halting problem has its origins in the paradoxes of self-reference. Gödel's theorem can be thought of as a mathematization of the Cretan liar paradox. The sixthcentury BC Cretan philosopher Epimenides is said to have declared that all Cretans are liars. (As Paul says of the Cretans in his letter to Titus 1:12: 'One of themselves, even a prophet of their own, said, The Cretians are alway liars, evil beasts, slow bellies. This witness is true.') More concisely, the fourth-century BC Miletan philosopher Eubulides, stated the paradox as "A man says, "What I am saying is a lie."' The paradox arises be- 
cause if the man is telling the truth, then he is lying, while if he is lying, then he is telling the truth.

Potential resolutions of this paradox had been discussed for more than two millenia by the time that Kurt Gödel constructed his mathematical elaboration of the paradox in 1931. Gödel developed a method for assigning a natural number or 'Gödel number' to each well-formed statement or formula in a mathematical theory or formal language. He then constructed theories that contained statements that were versions of the liar paradox ('this statement can not be proved to be true') and used Gödel numbering to prove that such a theory must be either inconsistent (false statements can be proved to be true) or incomplete: there exist statements that are true but that can not be proved to be true within the theory.

Gödel's original incompleteness theorems were based on formal language theory. Turing's contribution was to re-express these theorems in terms of a mechanistic theory of computation. The Turing machine is an abstract computing machine that is based on how a mathematician calculates. Turing noted that mathematicians think, write down formulae on sheets of paper, return to earlier sheets to look at and potentially change the formulae there, and pick up new sheets of paper on which to write. A Turing machine consists of a 'head,' a system with a finite number of discrete states that is the analogue to the mathematician, and a 'tape,' a set of squares each of which can either be blank or contain one of finite number of symbols, analogous to the mathematician's sheets of paper. The tape originally contains a finite number of non-blank squares, the program, which specify the computation to be performed. The head is prepared in a special 'start' state and placed at a specified square on the tape. Then as a function of the symbol on the square and of its own internal state, the head can alter the symbol, change its own internal state, and move to the square on the left or right. The machine starts out, and like a mathematician, the head reads symbols, changes them, moves on to different squares/sheets of paper, writes and erases symbols there, and moves on. The computation halts when the head enters a special 'stop' state, at which point the tape contains the output to the computation. If the head never enters the stop state, then computation never halts.

Turing's definition of an abstract computing machine is both simple and subtle. It is simple because the components (head and squares of tape) are all discrete and finite, and the computational dynamics are finitely specified. In fact, a Turing machine whose head has only two states, and whose squares have only three states can be universal in 
the sense that it can simulate any other Turing machine. The definition is subtle because the computation can potentially continue for ever, so that the number of squares of tape covered with symbols can increase without bound. One of the simplest questions that one can ask about a Turing machine is whether the machine, given a particular input, will ever halt and give an output, or whether it will continue to compute for ever. Turing showed that no Turing machine can compute the answer to this question for all Turing machines and for all inputs: the answer to the question of whether a given Turing machine will halt on a given input is uncomputable.

Like the proof Gödel's incompleteness theorems, Turing's proof of the halting problem relies on the capacity of Turing machines for self-reference. Because of their finite nature, one can construct a Gödel numbering for the set of Turing machines. Similarly, each input for a Turing machine can be mapped to a natural number. Turing showed that a Turing machine that computes whether a generic Turing machine halts given a particular input can not exist. The self-referential part of the proof consists of examining what happens when this putative Turing machine evaluates what happens when a Turing machine is given its own description as input. If such a Turing machine exists, then we can define another Turing machine that halts only if it doesn't halt, and fails to halt only if it halts, a computational analogue to the Cretan liar paradox (Saint Paul: 'damned slow-bellied Turing machines').

The only assumption that led to this self-contradiction is the existence of a Turing machine that calculates whether Turing machines halt or not. Accordingly, such a Turing machine does not exist: the question of whether a Turing machine halts or not given a particular input is uncomputable. This doesn't mean that one can't compute some of the time whether Turing machines halt or not: indeed, you can just let a particular Turing machine run, and see what happens. Sometimes it will halt and sometimes it won't. The fact that it hasn't halted yet even after a very long time makes it unlikely to halt, but it doesn't mean that it won't halt someday. To paraphrase Abraham Lincoln, you can tell whether some Turing machines halt all the time, and you can tell whether all Turing machines halt some of the time, but you can't tell when all Turing machines halt all of the time.

\section{The halting problem and the energy spectrum of physical systems}

At first it might seem that the halting problem, because of its abstract and paradoxical nature, might find little application in the description of physical systems. I will now show, 
to the contrary, that the halting problem arises in the computation of basic features of many physical systems. The key point to keep in mind is that even quite simple physical systems can be capable of universal digital computation. For example, the Ising model is perhaps the simplest model of a set of coupled spins, atoms, quantum dots, or general two-level quantum systems (qubits). Arbitrary logical circuits can effectively be written into the ground state of the inhomogeneous Ising model, showing that the Ising model is capable of universal computation in the limit that the number of spins goes to infinity [7]. When timevarying fields such as the electromagnetic field are added, the number of systems capable of universal computation expands significantly: almost any set of interacting quantum systems is capable of universal computation when subject to a global time-varying field [8].

The ubiquity of computing systems means that the answers to many physical questions are in fact uncomputable. One of the most useful questions that one can ask of a physical system is what are the possible values for the system's energy, i.e., what is the system's spectrum. A system's spectrum determines many if not most of the features of the system's dynamics and thermodynamics. In particular, an important question to ask is whether a system's spectrum is discrete, consisting of well-separated values for the energy, or continuous.

For example, if there is a gap between the lowest or 'ground state' energy and the next lowest or 'first excited state' energy, then the spectrum is discrete and the system is said to possess an energy gap. By contrast, if the spectrum is continuous in the vicinity of the ground state, then the system is said to be gapless. Gapped and gapless systems exhibit significantly different dynamic and thermodynamic behavior. In a gapped system, for example, the fundamental excitations or particles are massive, while in a gapless sytem they are massless. In a gapped system, the entropy goes to zero as the temperature goes to zero, while for a gapless system, the entropy remains finite, leading to significantly different thermodynamic behavior in the low temperature limit.

As will now be seen, if a physical system is capable of universal computation, the answer to the question of whether a particular part of its spectrum is discrete or continuous is uncomputable. In 1986, the Richard Feynman exhibited a simple quantum system whose whose dynamics encodes universal computation [9]. Feynman's system consists of a set of two-level quantum systems (qubits), coupled to a clock. The computation is encoded as a sequence of interactions between qubits. Every time an interaction is performed, the 
clock 'ticks' or increments by one. In a computation that halts, the computer starts in the initial program state and then explores a finite set of states. In a computation that doesn't halt, the clock keeps on ticking forever, and the computer explores an infinite set of states. In 1992, I showed that this feature of Feynman's quantum computer implied that halting programs correspond to discrete sectors of the system's energy spectrum, while non-halting programs correspond to continuous sectors of the system's spectrum [10]. In particular, when the system has been programmed to perform a particular computation, the question of whether its spectrum has a gap or not is equivalent to the question of whether the computer halts or not.

More precisely, a non-halting computer whose clock keeps ticking forever corresponds to a physical system that goes through a countably infinite sequence of distinguishable states. Such a system by necessity has a continuum of energy eigenstates. Qualitatively, the derivation of this continuum comes because the energy eigenstates are essentially the Fourier transform of the clock states. But the Fourier transform of a function over an infinite discrete set (labels of different clock states explored by the computation), is a function over a continuous, bounded set (energy eigenvalues). So for a non-halting program, the spectrum is continuous. Similarly, a halting computer that only explores a finite set of distinguishable states has a discrete spectrum, because the Fourier transform of a function over a finite, discrete set (labels of the clock states) is also a function over a finite discrete set (energy eigenvalues). So for a halting program, the spectrum is discrete. The reader is referred to [10-11] for the details of the derivation.

Although the derivation in [10] was given specifically for Feynman's quantum computer, the generic nature of the argument implies that the answer to the question of whether any quantum system capable of universal computation is gapped or gapless is generically uncomputable. If the computation never halts, then the system goes through a countably infinite sequence of computational states and the spectrum is continuous. If the computation halts then the system goes through a finite sequence of computational states and the spectrum is discrete. But since many if not most infinite quantum systems that evolve according to nonlinear interactions are capable of universal computation, this implies that uncomputability is ubiquitous in physical law.

\section{The theory of everything is uncomputable}

As just shown, relatively prosaic aspects of physical systems, such as whether a system has an energy gap or not, are uncomputable. As will now be seen, grander aspirations 
of physics are also uncomputable. In particular, one of the long term goals of elementary particle physics is to construct 'the theory of everything' - the most concise unified theoretical description of all the laws of physics, including the interactions between elementary particles, and gravitational interactions. But the most concise description of any set of physical laws is in general uncomputable.

The reason stems from the theory of algorithmic information [12-14]. The algorithmic information content of a string of bits is the length of the shortest computer program that can produce that string as output. In other words, the algorithmic information content of a bit string is the most concise description of that string that can be written in a particular computer language. The idea of algorithmic information was first defined by Solomonoff [12] in order to construct a computational version of Ockham's razor, which urges us to find the most parsimonious explanation for a given phenomenon. (William of Ockham: Numquam ponenda est pluralitas sine necessitate - plurality should never be posited without necessity, and Frustra fit per plura quod potest fieri per pauciora - it is futile to do with more things what be done with fewer.) Kolmogorov [13] and Chaitin [14] independently arrived at the concept of algorithmic information.

Algorithmic information is an elegant concept which makes precise the notion of the most concise description. In aiming for a theory of everything, physicists are trying to find the most concise description of the set of laws that govern our observed universe. The problem is that this most concise description is uncomputable. The uncomputability of algorithmic information stems from Berry's paradox. Like all the paradoxes discussed here, Berry's paradox arises from the capacity for self reference.

The English language can be used to specify numbers, e.g., 'the smallest natural number that can expressed as the sum of two distinct prime numbers.' Any natural number can be defined in English, and amongst all English specifications for a given number, there is some specification that has the smallest number of words. Berry's paradox can be expressed as the following phrase: 'the smallest natural number that requires more than twelve words to specify.' Does this phrase specify a number? If it does, then that number can be specified in twelve words, in contradiction to the statement that it cannot be specified in twelve words or less.

The theory of computation makes Berry's paradox precise. The shortest description of a number is given by the shortest program on a given universal Turing machine that produces that number as output. Suppose that algorithmic information is computable. 
Then there is some program for the same Turing machine which, given a number as input, outputs the length of the shortest program that produces that number. Suppose that this program that computes algorithmic information content has length $\ell$. Now look at the algorithmic version of Berry's phrase: 'The smallest number whose algorithmic information content is greater than $\ell$ plus a billion.' Does this phrase specify a number? If algorithmic information is computable, then the answer is Yes. A program can compute that number by going through all natural numbers in ascending order, and computing their algorithmic information content. When the program reaches the smallest number whose algorithmic information content is greater than $\ell$ plus a billion, it outputs this number and halts. The length of this program is the length of subroutine that computes algorithmic information content, i.e., $\ell$, plus the length of the additional code needed to check whether the algorithmic information content of a number is greater than $\ell$ plus a billion, and if not, increment the number and check again. But the length of this additional code is far less than a billion symbols, and so the length of the program to produce the number in the algorithmic version of Berry's phrase is far less than $\ell$ plus a billion. So the algorithmic information content of the number in Berry's phase is also less than $\ell$ plus a billion, in contradiction to the phrase itself. Paradox!

As in the halting problem, the apparent paradox can be resolved by the concept of uncomputability. The only assumption that went into the algorithmic version of Berry's argument was that algorithmic information content is computable. This assumption lead to a contradiction. By the principle of reductio ad absurdum, the only conclusion is that algorithmic information content is uncomputable. The shortest description of a natural number or bit string can not in general be computed.

The uncomputability of shortest descriptions holds for any bit string, including the hypothetical bit string that describes the physical theory of everything. Physical laws are mathematical specifications of the behavior of physical system: they provide formulae that tell how measurable quantities change over time. Such quantities include the position and momentum of a particle falling under the force of gravity, the strength of the electromagnetic field in the vicinity of a capacitor, the state of an electron in a hydrogen atom, etc. Physical laws consist of equations that govern the behavior of physical systems, and those equations in turn can be expressed algorithmically.

There is a subtlety here. Many physical laws describe the behavior of continuous quantities. Algorithmic representations of the equations governing continuous quantities 
necessarily involve a discretization: for example, using a finite number of bits to represent floating-point variables. The concept of computability can be extended to the computability of continuous quantities as well [15]. It is important to verify that the number of bits needed to approximate continuous behavior to a desired degree of accuracy remains finite. For the known laws of physics, such discrete approximations of continuous behavior seem to be adequate in the regimes to which those laws apply. The places where discrete approximation to continuous laws break down - e.g. the singularities in the center of black holes - are also the places where the laws themselves are thought to break down.

The uncomputability of algorithmic information content implies that the most concise expression of Maxwell's equations or the standard model for elementary particles is uncomputable. Since Maxwell's equations are already concise - they are frequently seen on the back of a tee shirt - few scientists are working on making them even more terse. (Note, however, that terser the expression of Maxwell's equations, the more difficult they are to 'unpack': in all fairness, to assess the algorithmic information content of a physical law, one should also count the length of the extra computer code needed to calculate the predictions of Maxwell's equations.) In cases where the underlying physical law that characterizes some phenomena is unknown, however, as is currently the case for high $T_{C}$ superconductivity, then uncomputability can be a thorny problem: finding even one concise theory, let alone the most concise one, could be uncomputable.

Uncomputability afflicts all sciences, not just physics. A simplified but useful picture of the goal of scientific research is that scientists obtain large amounts of data about the world via observation and experiment, and then try to find regularities and patterns in that data. But a regularity or pattern is nothing more or less than a method for compressing the data: if a particular pattern shows up in many places in a data set, then we can create a compressed version of the data by describing the pattern only once, and then specifying the different places that the pattern shows up. The most compressed version of the data is in some sense the ultimate scientific description. There is a sense in which the goal of all science is finding theories that provide ever more concise descriptions of data.

\section{Computational complexity and physical law}

What makes a good physical law? Being concise and easily expressed is only one criterion. A second criterion is that the predictions of the law are readily evaluated. If a law is concisely expressed but its predictions can only be revealed by a computation that takes the age of the universe, then the law is not very useful. Phrased in the language of 
computational complexity, if a physical law is expressed in terms of equations that predict the future given a description of the past, ideally those predictions can be obtained in time polynomial in the description of the past state. For example, the laws of classical mechanics and field theory are described in terms of ordinary and partial differential equations that are readily encoded and evaluated on a digital computer.

There is no guarantee that easily evaluated laws exist for all phenomena. For example, no classical computer algorithm currently exists that can predict the future behavior of a complex quantum system. The obstacle to efficient classical simulation of quantum systems are the counterintuitive aspects of quantum mechanics such as quantum superposition and entanglement which evidently require exponential amounts of memory space to represent on a classical computer. It seems to be as hard for classical computers to simulate quantum weirdness as it is for human beings to comprehend it.

Embryonic quantum computers exist, however, and are capable of simulating complex quantum systems in principle [16]. Indeed, the largest scale quantum computations to date have been performed by specialized quantum information processors that simulate different aspects of quantum systems. In [17] Cory simulated the propagation of spin waves in a sample of $O\left(10^{18}\right)$ fluorine spins. More recently, the D-Wave adiabatic quantum computer has found the ground state of Ising models using 512 superconducting quantum bits [18]. If we expand the set of computational devices that we use to define computational complexity, then the consequences of the known laws of physics can in principle be elaborated in polynomical time on classical and quantum computers.

If we restrict our attention to laws whose consequences can be evaluated in polynomial time, then the problem of finding concise expressions of physical laws is no longer uncomputable. The obstacle to finding the shortest program that produces a particular data string is essentially the halting problem. If we could tell that a program never halts, then we could find the shortest program to produce the data by going through all potential programs in ascending order, eliminating as we go along all non-halting programs from the set of programs that could potentially reproduce the data. That is, a halting oracle would allow us to find the shortest program in finite time.

In the case where we restrict our attention to programs that halt in time bounded by some polynomial in the length of the inputs, by contrast, if the program hasn't halted and reproduced the data by the prescribed time we eliminate it from consideration and move on to the next potential program. This construction shows that the problem of finding the 
shortest easily evaluated program is in the computational complexity class NP: one can check in polynomial time whether a given program will reproduce the data. Indeed, the problem of finding the shortest program to produce the data is NP-complete: it is a variant of the NP-complete problem of finding if there is any program within a specified subset of programs that gives a particular output. If we restrict our attention to physical laws whose predictions can be readily evaluated, then the problem of finding the most concise law to explain a particular data set is no longer uncomputable, it is merely NP complete. Probably exponentially hard is better than probably impossible, however.

Similarly, in the case of evaluating the energy gap of a physical system capable of computation, if the system runs for time $n$, then the gap can is no greater than $1 / n^{2}$. Accordingly, the problem of finding out whether the gap is smaller than some bound $\epsilon$ is also NP-hard.

\section{Discussion}

This chapter reviewed how uncomputability impacts the laws of physics. Although uncomputability as in the halting problem arises from seemingly esoteric logical paradoxes, I showed that common and basic questions in physics have answers that are uncomputable. Many physical systems are capable of universal computation: to solve the question of whether such a system has discrete or continuous spectrum in a particular regime, or whether it is gapped or gapless, requires one to solve the halting problem. At a more general level one can think of the all scientific laws as providing concise, easily unpacked descriptions of obserbational and experimental data. The problem of finding the most concise description of a data set is uncomputable in general, and the problem of finding the most concise description whose predictions are easily evaluated is NP-complete. Science is hard, and sometimes impossible. But that doesn't mean we shouldn't do it.

Acknowledgements: The author would like to thank Scott Aaronson for helpful discussions. 
References

[1] A.M. Turing Proc. London Math. Soc. 242 230265, 243 544-546 (1937).

[2] K. Gödel Monat. Math. Phys. 38, 173198 (1931).

[3] M. Cunningham, The History of Computation, AtlantiSoft, New York, 1997.

[4] C. Shannon, A Symbolic Analysis of Relay and Switching Circuits, MIT MS thesis. 1937.

[5] S. Lloyd, Phys. Rev. Lett. 75, 346-349, (1995).

[6] D. Deutsch, A. Barenco, A. Ekert, Proc. R. Soc. Lond. A 8, 669-677 (1995).

[7] F. Barahona, J. Phys. A 15, 3241 (1982).

[8] S. Lloyd, Science 261, 1569-1571 (1993).

[9] R.P. Feynman, Found. Phys. 16, 507-531 (1986).

[10] S. Lloyd, Phys. Rev. Lett. 71, 943-946 (1993).

[11] T. Cubitt et al., in preparation.

[12] R.J. Solomonoff, Inf. Cont., 7, 224-254 (1964).

[13] A.N. Kolmogorov, Prob. Inf. Trans., 1, 3-11 (1965).

[14] G.J. Chaitin, J. Ass. Comp. Mach. 13, 547-569 (1966).

[15] L. Blum, M. Shub, S. Smale, Bull. Am. Math. Soc. 21, 1-46 (1989).

[16] S. Lloyd, Science 273, 1073-1078, 1996.

[17] W. Zhang, D. Cory, Phys. Rev. Lett. 80, 1324-1347 (1998).

[18] M.W. Johnson et al. Nature 473 194-198 (2011). 